\title{
CDISC Events Class
}

National Cancer Institute

\section{Source}

National Cancer Institute. CDISC Events ClasS. NCI Thesaurus. Code C103372.

This SDT M class captures planned protocol milestones such as randomization and study completion, and occurrences, conditions, or incidents independent of planned study evaluations occurring during the trial (e.g., adverse events) or prior to the trial (e.g., medical history). 